\title{
CONSTRUCTING EQUIVARIANT HOMOTOPY EQUIVALENCES VIA EXTENSION OF SCALARS
}

\author{
MÁRIA ŠIMKOVÁ \\ (communicated by Donald M. Davis)
}

\begin{abstract}
Given a finite group $G$, we present a new algorithm to construct a $\mathbb{Z} G$-linear strong homotopy equivalence of $\mathbb{Z} G$ complexes from a $\mathbb{Z}$-linear one.
\end{abstract}

\section{Motivation}

A classical topological problem is to determine whether or not a given simplicial complex $K$ can be embedded into $\mathbb{R}^{d}$. Algorithmic aspects of this problem were studied in the paper [5]. Nevertheless, the question of whether or not the problem is algorithmically solvable remained open in the metastable range, i.e. $\operatorname{dim} K \leqslant \frac{2}{3} d-1$. In this range the problem is equivalent to the existence of a $\mathbb{Z} / 2$-equivariant map from the deleted product $(K \times K) \backslash \Delta_{K}$ into $S^{d-1}$ (see [8]). A more general problem has been solved in [1]. The authors considered two finite simplicial sets $X$ and $Y$ with a free action of a finite group $G$ such that $\operatorname{dim} X \leqslant 2 \operatorname{conn} Y+1$ and $\operatorname{conn} Y \geqslant 1$. In this case the set of homotopy classes of $G$-equivariant maps is an empty set or can be equipped with the structure of a finitely generated abelian group. The isomorphism type of this set can be determined algorithmically. The algorithm works with the equivariant Moore-Postnikov tower for a certain map $Y \rightarrow E G$ where $E G$ is an equivariant analogue of a point among free $G$-spaces. The stages of the tower are twisted products of $E G$ and Eilenberg-MacLane spaces which are generally infinite simplicial sets. To work with them algorithmically their associated non-degenerate chain complexes have to be strongly homotopy equivalent to locally finitely generated chain complexes equivariantly. In the so-called effective homological algebra introduced in $[\mathbf{6}]$ it is well-known that chain complexes associated to Eilenberg-MacLane spaces $K(\pi, n)$ (here $\pi$ is a finitely generated abelian group) and their twisted products with $E G$ are strongly homotopy equivalent to locally finite chain complexes but not equivariantly (see $[\mathbf{2}]$ ). The problem of construction of a suitable $\mathbb{Z} G$-homotopy equivalence has already been solved in [7] using homotopy algebras. Here we give a different algorithm based only on perturbation lemmas.

The research was supported by the project MUNI/A/1103/2016.

Received November 26, 2017, revised February 1, 2018; published on June 13, 2018.

2010 Mathematics Subject Classification: 18G10, 68W30.

Key words and phrases: strong homotopy equivalence, bar construction, perturbation lemma, effective homological algebra, chain complex, differential graded algebra.

Article available at http://dx.doi.org/10.4310/HHA.2018.v20.n2.a14

Copyright (C) 2018, International Press. Permission to copy for private use granted. 


\section{The main theorem}

\section{Notation}

The integral group algebra of a finite group $G$ will be denoted by $\mathbb{Z} G$. By abuse of notation a symbol $\mathbb{Z}$ will stand for a differential graded algebra $(\mathbb{Z}, 0)$ concentrated in dimension 0. A chain complex of free $\mathbb{Z} G$-modules will be called a $\mathbb{Z} G$-complex. The pair $(\mathcal{R}, d)$ will denote a differential non-negatively graded algebra (dga) over $\mathbb{Z}$ whose underlying chain complex is a $\mathbb{Z}$-complex with 1 as a generator. We say that a $\mathbb{Z} G$-complex is locally finite if it consists of finitely generated $\mathbb{Z} G$-modules.

We will work with differential graded modules over the dga $\mathcal{R}$ and we follow the approach of the paper [4, p. 832].

Definition 2.1. A (left) module over the dga $(\mathcal{R}, d)$ is a chain complex $\left(M, d_{M}\right)$ together with a linear map of degree zero $\cdot: \mathcal{R} \otimes_{\mathbb{Z}} M \rightarrow M$ defined as $r \otimes m \mapsto r \cdot m$ such that the following conditions hold for $1, r, r^{\prime} \in \mathcal{R}, m \in M$ :

- $\operatorname{associativity~}\left(r r^{\prime}\right) \cdot m=r \cdot\left(r^{\prime} \cdot m\right)$,

- identity element $1 \cdot m=m$,

- the Leibniz rule $d_{M}(r \cdot m)=d(r) \cdot m+(-1)^{|r|} r \cdot d_{M}(m)$.

An $\mathcal{R}$-linear map between $(\mathcal{R}, d)$-modules $\left(M, d_{M}\right)$ and $\left(N, d_{N}\right)$ of degree $k$ is a $\mathbb{Z}$ linear map $\varphi: M \rightarrow N$ of degree $k$ satisfying $\varphi(r \cdot m)=(-1)^{k|r|} r \cdot \varphi(m)$ for $r \in \mathcal{R}$ and $m \in M$. We say that the $(\mathcal{R}, d)$-module $\left(M, d_{M}\right)$ is free if it is free as a graded module over the graded algebra $\mathcal{R}$ i.e. $M \cong \mathcal{R} \otimes_{\mathbb{Z}} V$ with $V$ a free graded $\mathbb{Z}$-module. A free $(\mathcal{R}, d)$-module $\left(M, d_{M}\right)\left(M \cong \mathcal{R} \otimes_{\mathbb{Z}} V\right)$ is locally finite if the $\mathbb{Z}$-modules $V_{n}$ are finitely generated for all $n \in \mathbb{Z}$.

Remark 2.2. We note that a $\mathbb{Z} G$-complex is a free differential graded ( $\mathbb{Z} G, 0)$-module.

\section{Effective homological algebra}

To state the main theorem we need to recall some basic notions of effective homological algebra (see $[\mathbf{6}])$ for $(\mathcal{R}, d)$-modules.

Definition 2.3 ([6, Definition 47$])$. Let $\left(C, d_{C}\right)$ and $\left(D, d_{D}\right)$ be $(\mathcal{R}, d)$-modules. A triple of mappings $(f: C \rightarrow D, g: D \rightarrow C, h: C \rightarrow C)$ is called a reduction if the following conditions hold:

- $f$ and $g$ are $\mathcal{R}$-linear chain maps of degree 0 ,

- $h$ is an $\mathcal{R}$-linear map of degree 1 ,

- $f g=\operatorname{id}_{D}$ and $\operatorname{id}_{C}-g f=\left[d_{C}, h\right]=d_{C} h-h d_{C}$,

- $f h=0, h g=0$ and $h h=0$.

The reductions are denoted by $(f, g, h):\left(C, d_{C}\right) \Rightarrow\left(D, d_{D}\right)$.

Definition 2.4 ([6, Definition 53]). A strong homotopy equivalence $C \Leftrightarrow D$ between $(\mathcal{R}, d)$-modules $C, D$ is an $(\mathcal{R}, d)$-module $E$ and a pair of reductions $C \Leftarrow E \Rightarrow D$.

Definition 2.5 ([7, pp. 3-4]). An $(\mathcal{R}, d)$-module is locally effective if

- elements of the graded module can be represented in a computer,

- the operations of zero, addition, differential and multiplication by elements of $\mathcal{R}$ are computable. 
A locally effective $(\mathcal{R}, d)$-module is free if an arbitrary element of the underlying $(\mathcal{R}, d)$-module is represented in a computer as an $\mathcal{R}$-linear combination of its distinguished basis. A map is locally effective if there is an algorithm computing its values.

The previous definition can be illustrated by the following example.

Example 2.6. Consider a simplicial set $X$ with a free action of a finite group $G$ and its associated normalized chain complex $C_{*}(X)$. The distinguished $\mathbb{Z}$-basis of $C_{*}(X)$ consists of non-degenerate simplices. If there is a free simplicial $G$-action defined on the simplicial set $X$ then the chain complex $C_{*}(X)$ has induced free $G$-action by chain maps. Thus the distinguished $\mathbb{Z} G$-basis of $C_{*}(X)$ is formed by representatives of $G$-orbits.

More detailed descriptions of the above terminology can be found in [1, p. 9].

Convention 2.7. We assume that all $(\mathcal{R}, d)$-modules and maps are locally effective.

The aim of this paper is to prove the following statement.

Theorem 2.8. Let $M$ be a locally effective $\mathbb{Z} G$-complex and let $N$ be a locally effective and locally finite $\mathbb{Z}$-complex. Given a locally effective strong homotopy equivalence of $\mathbb{Z}$-complexes $M \Leftrightarrow N$, there is an algorithm which constructs a locally effective and locally finite $\mathbb{Z} G$-complex $N^{\prime}$ and a locally effective $\mathbb{Z} G$-linear strong homotopy equivalence $M \Leftrightarrow N^{\prime}$.

So the main statement will be the consequence of the following more general theorem if we put $\mathcal{R}=\mathbb{Z} G$.

Theorem 2.9. Let $\mathcal{R}$ be a differential non-negatively graded algebra over $\mathbb{Z}$ whose underlying chain complex is a $\mathbb{Z}$-complex with 1 as a generator. Let $M$ be a free locally effective $(\mathcal{R}, d)$-module and let $N$ be a locally effective and locally finite $\mathbb{Z}$-complex. Given a locally effective strong homotopy equivalence of $\mathbb{Z}$-complexes $M \Leftrightarrow N$, there is an algorithm which constructs a free locally effective and locally finite $(\mathcal{R}, d)$-module $N^{\prime}$ and a locally effective $\mathcal{R}$-linear strong homotopy equivalence $M \Leftrightarrow N^{\prime}$.

\section{Outline}

We start in the same way as in [7] and we will construct $(\mathcal{R}, d)$-modules and an $\mathcal{R}$ linear strong homotopy equivalence $B(\mathcal{R}, \mathcal{R}) \otimes_{\mathbb{Z}} M \Leftrightarrow B(\mathcal{R}, \mathcal{R}) \otimes_{\mathbb{Z}} N$ where $B(\mathcal{R}, \mathcal{R})$ is the bar construction on the dga $\mathcal{R}$. Then the differential of the bar construction on the dga $\mathcal{R}$ with coefficients in $M$, denoted by $B(\mathcal{R}, \mathcal{R}, M)$, is obtained from the differential of $B(\mathcal{R}, \mathcal{R}) \otimes_{\mathbb{Z}} M$ by a perturbation. This perturbation can be transferred using the strong homotopy equivalence on the $(\mathcal{R}, d)$-module $B(\mathcal{R}, \mathcal{R}) \otimes_{\mathbb{Z}} N$. The resulting perturbated $(\mathcal{R}, d)$-module $N^{\prime}$ is strongly homotopy equivalent to $B(\mathcal{R}, \mathcal{R}, M)$ and using a natural reduction $B(\mathcal{R}, \mathcal{R}, M) \Rightarrow M$ we get

$$
M \Leftarrow B(\mathcal{R}, \mathcal{R}, M) \Leftrightarrow N^{\prime} .
$$

\section{Overview of basic notions}

In this section we will summarize some fundamental results in homological algebra which will form the background of the last section. The results are adapted from the paper $[\mathbf{6}]$ and will be reformulated for $(\mathcal{R}, d)$-modules. 
Definition 3.1 ([6, Definition 49]). Let $\left(C, d_{C}\right)$ be an $(\mathcal{R}, d)$-module. An $\mathcal{R}$-linear mapping $\delta_{C}$ of degree -1 is a perturbation of the differential $d_{C}$ if $\left(d_{C}+\delta_{C}\right)^{2}=0$. On the chain complex $\left(C, d_{C}+\delta_{C}\right)$ we consider the same multiplication by elements of $\mathcal{R}$ as in $\left(C, d_{C}\right)$. Then $d_{C}+\delta_{C}$ satisfies the Leibniz rule since

$$
\begin{aligned}
\left(d_{C}+\delta_{C}\right)(r \cdot x) & =d_{C}(r \cdot x)+\delta_{C}(r \cdot x) \\
& =d(r) \cdot x+(-1)^{|r|} r \cdot d_{C}(x)+(-1)^{|r|} r \delta_{C}(x) \\
& =d(r) \cdot x+(-1)^{|r|} r \cdot\left(d_{C}+\delta_{C}\right)(x) .
\end{aligned}
$$

Hence $\left(C, d_{C}+\delta_{C}\right)$ is again a module over dga $(\mathcal{R}, d)$.

Proposition 3.2 (Basic perturbation lemma). Let $(f, g, h):\left(C, d_{C}\right) \Rightarrow\left(D, d_{D}\right)$ be a reduction between $(\mathcal{R}, d)$-modules and let $\delta_{C}$ be a perturbation of the differential $d_{C}$ of the $(\mathcal{R}, d)$-module $C$. Assume that the following nilpotency condition is satisfied: for all $c \in C$, there exists $\nu \in \mathbb{N}_{0}$ satisfying $\left(h \delta_{C}\right)^{\nu}(c)=0$. Then there exists a perturbation $\delta_{D}$ of the differential $d_{D}$ and an $\mathcal{R}$-linear reduction $\left(f^{\prime}, g^{\prime}, h^{\prime}\right):\left(C, d_{C}+\delta_{C}\right) \Rightarrow$ $\left(D, d_{D}+\delta_{D}\right)$ where $\left(C, d_{C}+\delta_{C}\right)$ and $\left(D, d_{D}+\delta_{D}\right)$ have the canonical $(\mathcal{R}, d)$-module structure described in Definition 3.1.

Proof. The $\mathcal{R}$-linearity of the maps $\left(f^{\prime}, g^{\prime}, h^{\prime}\right)$ and $\delta_{D}$ directly follows from their definitions

$$
\begin{aligned}
f^{\prime} & =f \circ \sum_{i=0}^{\infty}(-1)^{i}\left(\delta_{C} h\right)^{i}, \\
g^{\prime} & =\sum_{i=0}^{\infty}(-1)^{i}\left(h \delta_{C}\right)^{i} \circ g, \\
h^{\prime} & =\sum_{i=0}^{\infty}(-1)^{i}\left(h \delta_{C}\right)^{i} \circ h, \\
\delta_{D} & =f \circ \delta_{C} \circ g^{\prime} .
\end{aligned}
$$

For the rest of this proof we refer to [6, Theorem 51].

The proof of the following "Easy perturbation lemma" is based on a similar statement for $(\mathbb{Z}, 0)$-modules (see [6, Proposition 50]).

Proposition 3.3 (Easy perturbation lemma). Let $(f, g, h):\left(C, d_{C}\right) \Rightarrow\left(D, d_{D}\right)$ be a reduction between $(\mathcal{R}, d)$-modules and let $\delta_{D}$ be a perturbation of the differential $d_{D}$ of the $(\mathcal{R}, d)$-module $D$. Then there is a perturbated reduction $(f, g, h):\left(C, d_{C}+\right.$ $\left.\delta_{C}\right) \Rightarrow\left(D, d_{D}+\delta_{D}\right)$ where the perturbation of the differential $d_{C}$ is defined as $\delta_{C}=$ $g \delta_{D} f$. The $(\mathcal{R}, d)$-module structures of $\left(C, d_{C}+\delta_{C}\right)$ and $\left(D, d_{D}+\delta_{D}\right)$ are canonical as described in Definition 3.1.

Proposition 3.4 ([6, Proposition 60]). Given two reductions $(f, g, h): C \Rightarrow D$ and $\left(f^{\prime}, g^{\prime}, h^{\prime}\right): D \Rightarrow E$ between $(\mathcal{R}, d)$-modules, there is a composition of the reductions $\left(f^{\prime} f, g g^{\prime}, h+g h^{\prime} f\right): C \Rightarrow E$.

Proposition 3.5 ([6, Proposition 61]). Given two reductions $(f, g, h): C \Rightarrow \widetilde{C}$ and $\left(f^{\prime}, g^{\prime}, h^{\prime}\right): D \Rightarrow \widetilde{D}$ between $(\mathbb{Z}, 0)$-modules, there is a reduction $\left(f \otimes f^{\prime}, g \otimes g^{\prime}, h \otimes\right.$ $\left.\operatorname{id}_{D}+(g \circ f) \otimes h^{\prime}\right): C \otimes_{\mathbb{Z}} D \rightarrow \widetilde{C} \otimes_{\mathbb{Z}} \widetilde{D}$ which is called a tensor product of reductions. 


\section{The bar construction}

For the proof of our main result we utilize the bar construction $B(\mathcal{R}, \mathcal{R})$ as described in $[4$, p. 845] and its differentials in [7, p. 12]. We recall that the suspension of the chain complex $(\mathcal{R}, d)$ will be denoted by $(s \mathcal{R},-d)$ and defined by $(s \mathcal{R})_{n}=$ $(\mathcal{R})_{n-1}$ for $n \in \mathbb{N}$. The tensor algebra of $s \mathcal{R}$ is defined as $T(s \mathcal{R})=\bigoplus_{k=0}^{\infty}(s \mathcal{R})^{\otimes k}$ and we define $(s \mathcal{R})^{\otimes 0}=\mathbb{Z}$.

Firstly, we define a $(\mathbb{Z}, 0)$-module $B(\mathcal{R}, \mathcal{R})=\mathcal{R} \otimes_{\mathbb{Z}} T(s \mathcal{R})$ with a differential $d_{B}$. The generators of $B(\mathcal{R}, \mathcal{R})$ will be denoted by $r_{0}\left|r_{1}\right| \cdots \mid r_{k}$. According to Eilenberg and Mac Lane's terminology [3, p. 73$]$ the number $k$ is called the simplicial degree and the sum $|r|_{0,1, \ldots, k}=\left|r_{0}\right|+\left|r_{1}\right|+\cdots+\left|r_{k}\right|$ is the tensor degree of the generator $r_{0}\left|r_{1}\right| \cdots \mid r_{k}$. Moreover, we put $|r|_{-1}=0$. The differential $d_{B}$ is defined as follows

$$
\begin{aligned}
d_{B}\left(r_{0}\left|r_{1}\right| \cdots \mid r_{k}\right) & =\sum_{i=0}^{k} d_{i}^{\otimes}\left(r_{0}\left|r_{1}\right| \cdots \mid r_{k}\right)+\sum_{m=1}^{k} d_{m}^{\text {alg }}\left(r_{0}\left|r_{1}\right| \cdots \mid r_{k}\right), \\
d_{i}^{\otimes}\left(r_{0}\left|r_{1}\right| \cdots \mid r_{k}\right) & =(-1)^{i+|r|_{0,1, \ldots, i-1}} r_{0}\left|r_{1}\right| \cdots\left|d\left(r_{i}\right)\right| \cdots \mid r_{k}, \\
d_{m}^{\text {alg }}\left(r_{0}\left|r_{1}\right| \cdots \mid r_{k}\right) & =(-1)^{m-1+|r|_{0,1, \ldots, m-1}} r_{0}\left|r_{1}\right| \cdots\left|r_{m-1} r_{m}\right| \cdots \mid r_{k} .
\end{aligned}
$$

It is easy to verify that equation (1) defines a differential i.e. $d_{B}^{2}=0$. More details can be found in $[\mathbf{3}$, Chapter 2]. The module $B(\mathcal{R}, \mathcal{R})$ is usually called the bar construction on the dga $\mathcal{R}$. We note that $B(\mathcal{R}, \mathcal{R})$ is an $(\mathcal{R}, d)$-module with multiplication

$$
r \cdot\left(r_{0}\left|r_{1}\right| \cdots \mid r_{k}\right)=\left(r \cdot r_{0}\right)\left|r_{1}\right| \cdots \mid r_{k}
$$

More generally, we define an $(\mathcal{R}, d)$-module $\left(B(\mathcal{R}, \mathcal{R}, M), \partial_{M}+\delta_{M}\right)$ for a given $(\mathcal{R}, d)$-module $\left(M, d_{M}\right)$ in the following way (we use the Koszul sign convention)

$$
\begin{aligned}
B(\mathcal{R}, \mathcal{R}, M)= & \mathcal{R} \otimes_{\mathbb{Z}} T(s \mathcal{R}) \otimes_{\mathbb{Z}} M \\
\partial_{M}\left(r_{0}\left|r_{1}\right| \cdots \mid r_{k} \otimes x\right)= & \left(d_{B} \otimes_{\mathbb{Z}} \operatorname{id}_{M}\right)\left(r_{0}\left|r_{1}\right| \cdots \mid r_{k} \otimes x\right)+ \\
& +\left(\operatorname{id}_{B(\mathcal{R}, \mathcal{R})} \otimes_{\mathbb{Z}} d_{M}\right)\left(r_{0}\left|r_{1}\right| \cdots \mid r_{k} \otimes x\right), \\
\delta_{M}\left(r_{0}\left|r_{1}\right| \cdots \mid r_{k} \otimes x\right)= & \begin{cases}(-1)^{k+|r|_{0,1, \ldots, k-1}} r_{0}\left|r_{1}\right| \cdots \mid r_{k-1} \otimes r_{k} x & \text { for } k>0, \\
0 & \text { for } k=0,\end{cases}
\end{aligned}
$$

where the differential $d_{B}$ is defined in (1). Similarly as before, we need to show that $\left(\partial_{M}+\delta_{M}\right)^{2}=0$ which also follows from Lemma 9 in $[\mathbf{7}$, p. 12]. The module $B(\mathcal{R}, \mathcal{R}, M)$ is called the bar construction on the dga $\mathcal{R}$ with coefficients in $M$. There is an augmentation map $\epsilon: B(\mathcal{R}, \mathcal{R}, M) \rightarrow M$ which is given by

$$
\begin{aligned}
\epsilon\left(r_{0} \otimes x\right) & =r_{0} \cdot x, \\
\epsilon\left(r_{0}\left|r_{1}\right| \cdots \mid r_{k} \otimes x\right) & =0 \text { for } k>0 .
\end{aligned}
$$

Remark 4.1. The $(\mathcal{R}, d)$-module $B(\mathcal{R}, \mathcal{R}, M)$ is exactly $B(\mathcal{R}, \mathcal{R}) \otimes_{\mathbb{Z}} M$ with the differential $\partial_{M}$ perturbated by $\delta_{M}$.

At the end of this section we recall a natural reduction which we will use in the last section.

Theorem 4.2. Let $\mathcal{R}$ be a differential non-negatively graded algebra over $\mathbb{Z}$ whose underlying chain complex is a $\mathbb{Z}$-complex with 1 as a generator and let $M$ be an 
$(\mathcal{R}, d)$-module which is free as a graded module. Then the augmentation map

$$
\epsilon:\left(B(\mathcal{R}, \mathcal{R}, M), \partial_{M}+\delta_{M}\right) \rightarrow M
$$

is a projection of an $\mathcal{R}$-linear reduction.

Proof. See Theorem 4 in [7, p. 7].

\section{Construction of the $\mathcal{R}$-linear strong homotopy equivalence}

In this section we construct the required strong homotopy equivalence i.e. we will give a proof of Theorem 2.9. We start with a $\mathbb{Z}$-linear pair of reductions

$$
M \Leftarrow_{(f, g, h)} \widetilde{M} \Rightarrow_{\left(f^{\prime}, g^{\prime}, h^{\prime}\right)} N .
$$

Here $M$ is a free $(\mathcal{R}, d)$-module, $N$ and $\widetilde{M}$ are $(\mathbb{Z}, 0)$-modules. Proposition 3.5 can be applied to get $\mathbb{Z}$-linear reductions

$$
B(\mathcal{R}, \mathcal{R}) \otimes_{\mathbb{Z}} M \Leftarrow_{(\mathrm{id} \otimes f, \mathrm{id} \otimes g, \mathrm{id} \otimes h)} B(\mathcal{R}, \mathcal{R}) \otimes_{\mathbb{Z}} \widetilde{M} \Rightarrow_{\left(\mathrm{id} \otimes f^{\prime}, \mathrm{id} \otimes g^{\prime}, \mathrm{id} \otimes h^{\prime}\right)} B(\mathcal{R}, \mathcal{R}) \otimes_{\mathbb{Z}} N .
$$

Since these modules are $(\mathcal{R}, d)$-modules and the identity on $B(\mathcal{R}, \mathcal{R})$ is $\mathcal{R}$-linear, the reductions in (4) are $\mathcal{R}$-linear.

Note that the differentials of $(\mathcal{R}, d)$-modules in (4) are given by the equation $(2)$. We apply the Easy perturbation lemma (Proposition 3.3) to the reduction (id $\otimes f$, id $\otimes g, \mathrm{id} \otimes h)$ and the perturbation $\delta_{M}$ of the $(\mathcal{R}, d)$-module $B(\mathcal{R}, \mathcal{R}) \otimes_{\mathbb{Z}} M$ from $(3)$. The resulting perturbation of the $(\mathcal{R}, d)$-module $B(\mathcal{R}, \mathcal{R}) \otimes_{\mathbb{Z}} \widetilde{M}$ is

$$
\delta_{\widetilde{M}}=(\mathrm{id} \otimes g) \delta_{M}(\mathrm{id} \otimes f) .
$$

Now we are going to apply the Basic perturbation lemma (Proposition 3.2) to the reduction $\left(\mathrm{id} \otimes f^{\prime}, \mathrm{id} \otimes g^{\prime}, \mathrm{id} \otimes h^{\prime}\right)$ and the perturbation $\delta_{\widetilde{M}}$. We have to prove that the nilpotency condition is satisfied for all $c \in B(\mathcal{R}, \mathcal{R}) \otimes_{\mathbb{Z}} \widetilde{M}$. The main idea is based on the fact that the perturbation

$$
\delta_{\widetilde{M}}: \mathcal{R} \otimes_{\mathbb{Z}}(s \mathcal{R})^{k} \otimes_{\mathbb{Z}} \widetilde{M} \rightarrow \mathcal{R} \otimes_{\mathbb{Z}}(s \mathcal{R})^{k-1} \otimes_{\mathbb{Z}} \widetilde{M}
$$

decreases the simplicial degree $k$, if $k>0$, and for $k=0$

$$
\delta_{\widetilde{M}}(r \otimes \widetilde{m})=(i d \otimes g) \delta_{M}(r \otimes f(\widetilde{m}))=0
$$

according to (3). We note that the map id $\otimes h^{\prime}$ does not have any impact on the simplicial degree. To demonstrate the previous statement we provide the explicit computation for $c=r_{0}\left|r_{1}\right| \cdots \mid r_{k} \otimes \widetilde{m}$. Using (3) we obtain:

$$
\begin{aligned}
\left(\left(\mathrm{id} \otimes h^{\prime}\right) \delta_{\widetilde{M}}\right)^{k+1}(c) & =\left(\left(\mathrm{id} \otimes h^{\prime}\right) \delta_{\widetilde{M}}\right)^{k}\left(\mathrm{id} \otimes\left(h^{\prime} g\right)\right) \delta_{M}(\mathrm{id} \otimes f)\left(r_{0}\left|r_{1}\right| \cdots \mid r_{k} \otimes \widetilde{m}\right) \\
& =\left(\left(\mathrm{id} \otimes h^{\prime}\right) \delta_{\widetilde{M}}\right)^{k}\left(\mathrm{id} \otimes\left(h^{\prime} g\right)\right) \delta_{M}\left(r_{0}\left|r_{1}\right| \cdots \mid r_{k} \otimes f(\widetilde{m})\right) \\
& = \pm\left(\left(\mathrm{id} \otimes h^{\prime}\right) \delta_{\widetilde{M}}\right)^{k}\left(\mathrm{id} \otimes\left(h^{\prime} g\right)\right)\left(r_{0}\left|r_{1}\right| \cdots \mid r_{k-1} \otimes r_{k} \cdot f(\widetilde{m})\right) \\
& = \pm\left(\left(\mathrm{id} \otimes h^{\prime}\right) \delta_{\widetilde{M}}\right)^{k}\left(r_{0}\left|r_{1}\right| \cdots \mid r_{k-1} \otimes h^{\prime}\left(g\left(r_{k} \cdot f(\widetilde{m})\right)\right)\right) \\
& = \pm\left(\mathrm{id} \otimes h^{\prime}\right) \delta_{\widetilde{M}}\left(r_{0} \otimes h^{\prime}\left(g\left(r_{1} \cdot f\left(\cdots\left(h^{\prime}\left(g\left(r_{k} \cdot f(\widetilde{m})\right)\right)\right) \cdots\right)\right)\right)\right) \\
& =0 .
\end{aligned}
$$

We denote the perturbated $(\mathcal{R}, d)$-module $\left(B(\mathcal{R}, \mathcal{R}) \otimes_{\mathbb{Z}} N, \partial_{N}+\delta_{N}\right)$ by $N^{\prime}$. Now we 
use Theorem 4.2 to obtain

$$
M \Leftarrow B(\mathcal{R}, \mathcal{R}, M) \Leftrightarrow N^{\prime} .
$$

Finally, the application of Proposition 3.4 yields the strong homotopy equivalence of $(\mathcal{R}, d)$-modules

$$
M \Leftrightarrow N^{\prime}
$$

If $N$ is a locally finite $\mathbb{Z}$-complex then $N^{\prime}$ is a free locally finite $(\mathcal{R}, d)$-module; this directly follows from its definition.

\section{Acknowledgments}

I would like to thank the anonymous referee for helpful suggestions and a lot of valuable comments which have contributed significantly to improving the article.

\section{References}

[1] M. Čadek, M. Krčál, L. Vokřínek. Algorithmic solvability of the lifting-extension problem. Discrete Comput. Geom., 57 (2017), no. 4, 915-965. ISSN 0179-5376. doi:10.1007/s00454-016-9855-6.

[2] M. Čadek, M. Krčál, J. Matoušek, L. Vokřínek, U. Wagner. Polynomial-time computation of homotopy groups and Postnikov systems in fixed dimension. SIAM J. Comput., 43 (2014), no. 5, 1728-1780. ISSN 0097-5397. doi:10.1137/ 120899029.

[3] S. Eilenberg, S. Mac Lane. On the groups $H(\pi, n)$ I. Ann. of Math. (2), 58 (1953), no. 1, 55-106. doi:10.2307/1969820.

[4] Y. Félix, S. Halperin, J-C. Thomas. Differential graded algebras in topology. Handbook of Algebraic Topology, 829-865, North-Holland, Amsterdam, 1995.

[5] J. Matoušek, M. Tancer, U. Wagner. Hardness of embedding simplicial complexes in $\mathbb{R}^{d}$. J. Eur. Math. Soc. (JEMS), 13 (2011), 259-295.

[6] J. Rubio, F. Sergeraert. Constructive homological algebra and applications. Preprint, arXiv:1208.3816 (2013).

[7] L. Vokřínek. Constructing homotopy equivalences of chain complexes of free $\mathbb{Z} G$ modules. An Alpine Expedition through Algebraic Topology, 279-296, Contemp. Math., 617, Amer. Math. Soc., Providence, RI, 2014.

[8] C. Weber. Plongements de polyedres dans le domaine metastable. Comment. Math. Helv., 42 (1967), 1-27.

Mária Šimková xsimkovam@math.muni.cz

Department of Mathematics and Statistics, Faculty of Science, Masaryk University, Kotlářská 2, Brno, 611 37, Czech Republic 